\title{
Persistent chorea as a manifestation of thyrotoxicosis
}

\author{
A. Javaid and D.D. Hilton \\ Corbett Hospital, Stourbridge, West Midlands DY8 4JB, UK.
}

\begin{abstract}
Summary: We report a case of persistent chorea as a manifestation of thyrotoxicosis. The chorea was severe and persisted after the patient was rendered euthyroid. Dopamine antagonists only partially suppressed the involuntary movements during the first few months. It was eventually controlled with haloperidol, but whenever she discontinues the treatment the chorea has returned during the 16 months since she first presented.
\end{abstract}

\section{Introduction}

Choreoathetosis is a recognized manifestation of thyrotoxicosis. It was first described by Sir William Gowers ${ }^{1}$ in 1893 . There have been several papers in the past reporting cases of chorea due to thyrotoxicosis. $^{2-6}$ In all these reports, except one, chorea was quickly reversible by propranolol, dopamine antagonists or anti-thyroid drugs. There is one case in the literature ${ }^{7}$ which describes chorea which dated from an attack of thyrotoxicosis 30 years earlier.

This paper reports a case of severe choreiform movements, with generalized hypotonia associated with thyrotoxicosis. The involuntary movements continued after the patient was rendered euthyroid. This case suggests that the neurological disorder following hyperthyroidism, which usually is transient, may become persistent.

\section{Case report}

A 15 year old girl was admitted to hospital as an emergency with violent involuntary movements. These movements had started 3 weeks earlier and gradually became worse, involving the whole body. She was dropping things at home and school. Shortly before admission the movements became increasingly violent and she collapsed at a friend's party and was unable to stand again.

Until the age of 11 she was short and fat, but then grew rapidly, becoming tall and thin. She grew 15 inches in 2 years and lost weight in spite of a voracious appetite. She also complained of being hot, sweaty and thirsty.

On examination she had violent choreiform

Correspondence: A. Javaid, M.R.C.P., Department of Respiratory Medicine, East Birmingham Hospital, Bordesley Green East, Birmingham B9 5ST, UK.

Accepted: 19 May 1988 movements of her arms, legs, neck and trunk. They were so severe that she had to be nursed in a hammock bed. There was profound generalized hypotonia with dysarthria and dysphagia. She had a tachycardia of 110 per minute, with blood pressure $145 / 70 \mathrm{~mm} \mathrm{Hg}$. There was a soft systolic murmur at the left sternal edge. She had a diffuse goitre. There were no occular signs of thyrotoxicosis.

The haematological investigation revealed hypochromic microcytic anaemia due to iron deficiency for which no cause was found. Serum electrolytes, renal function, liver function tests and copper studies were normal. No abnormality was found on skull X-ray or computed tomographic scan of the brain. Baseline serum growth hormone and its response to glucose load was normal.

Thyroid function tests showed that she was thyrotoxic with serum free thyroxine $\left(\mathrm{T}_{4}\right) 86.5 \mathrm{pmol} / 1$ (normal range 11-25), serum free triiodothyronine $\left(\mathrm{T}_{3}\right) \quad 18.7 \mathrm{pmol} / \mathrm{l}$ (normal range 3-9), thyroidstimulating hormone $0.05 \mathrm{mU} / 1$ (normal range 0.3 5.0). Thyroglobulin antibodies were positive $1: 160$ and microsomal antibodies positive 1:100. Streptococcal origin of chorea was excluded by negative throat swabs and normal anti-streptolysin and antihyaluronidase antibodies which were performed on two separate occasions. Anti-DNA antibody tests were also negative. Other bacteriological investigations were also normal and so were the viral complement fixation tests and spirochaetal serology.

As soon as the diagnosis of thyrotoxicosis was made she was started on propranolol $40 \mathrm{mg}$ t.d.s. and carbimazole $20 \mathrm{mg}$ t.d.s. Symptomatic treatment for the chorea was started with chlorpromazine $25 \mathrm{mg}$ t.d.s. The dose was increased and later tetrabenazine $25 \mathrm{mg}$ t.d.s. was added, the dose of which was also increased to $50 \mathrm{mg}$ t.d.s. The thyro- 
toxicosis responded to carbimazole and within 3 weeks she was euthyroid with normal serum free $T_{4}$ and $T_{3}$, but the chorea proved very refractory to treatment. There was no response to propranolol. The dopamine antagonists chlorpromazine and tetrabenazine only partially suppressed the movements. Over 3 months the chorea slowly improved and we attributed this to the treatment of her thyrotoxicosis. Six months later the chorea was still persisting. A further trial with a third dopamine antagonist, haloperidol, was made at this time which almost completely abolished the involuntary movements. The chorea has returned whenever she stopped taking the haloperidol. The most recent recurrence was 16 months after she first presented.

\section{Discussion}

Chorea is associated with a variety of disease states, indicating that there are several factors of importance in the pathophysiology of this extrapyramidal syndrome. In Huntington's chorea neuronal damage is found in the striatum ${ }^{8}$ whereas the chorea in thyrotoxicosis is considered to be biochemical rather than due to structural damage. This is borne out by the usually rapid reversibility of chorea in thyrotoxicosis and by the results of a post-mortem quoted in Waldenstram's review. ${ }^{7}$

In all forms of chorea it has been observed that dopamine agonists produce an exacerbation while dopamine antagonists ameliorate the condition. The mechanism of chorea due to thyrotoxicosis is thought to be due to increased sensitivity of the dopaminergic receptor sites in the corpus striatum. Alterations in thyroid function have been found to

\section{References}

1. Gowers, W.R. A Manual of Disease of the Nervous System, 2nd edition. J.A. Churchill, London, 1893.

2. Sutherland, G.A. Chorea and Graves' disease. Brain 1903, 26: 210 .

3. Syner, J.C., Fencher, P.S. \& Kemble, J.W. Chorea associated with hyperthyroidism. US Armed Forces Med J 1945, 5: 61-66.

4. Fidler, S.M., O'Rourke, R.A. \& Bucksbaum, H.W. Chorio-athetosis as a manifestation of thyrotoxicosis. Neurology 1971, 21: 55-57.

5. Van Uitern, R.L. \& Rusakoff, L.M. Hyperthyroid chorea mimicking psychiatric disease. Am J Psychiatry 1979, 136: 1208-1211.

6. Delwaide, P.F. \& Shoenen, J. Hyperthyroidism as a cause of persistent choreic movements. Acta Neurol Scand 1978, 58: 309-312. influence the sensitivity of adrenergic and dopaminergic receptors to noradrenaline and dopamine. ${ }^{9}$ Klawans and Shenker ${ }^{10}$ have also shown that dopamine turnover is reduced in hyperthyroid patients as a reflection of hypersensitivity of dopaminergic receptors. Case reports of thyrotoxic chorea $^{2,4,10}$ have noted rapid response of the chorea to beta blockade, reserpine, dopamine antagonists or antithyroid drugs. The chorea did not return after the patients had been made euthyroid even when the beta-blockers, reserpine or dopamine antagonists were stopped.

Our observation and the case described by Delwaide and Shoenen ${ }^{6}$ are exceptions to the above rule. In our case the chorea was very severe and the hypotonia profound. Initially it was only partially responsive to symptomatic treatment. However, after the patient was rendered euthyroid, a small dose of haloperidol was still necessary to control the chorea.

We do not think that there was any cause for the chorea other than thyrotoxicosis. In particular, the negative tests for streptococci make Sydenham's chorea unlikely.

We would agree with Delwaide and Shoenen? that persistent chorea can be caused by thyrotoxicosis though we do not know of the factors which may make this condition a permanent one. It may be that some neurotoxic effects supervene to cause permanent damage. As Delwaide and Shoenen suggest, this may be akin to tardive dyskinesia which persists after treatment with phenothiazines has been stopped. The long history and severity of thyrotoxicosis may well have contributed to the permanent damage.

7. Waldenstram, J. Acute thyrotoxic encephalomyopathy or myopathy, its cause and treatment. Acta Micl Scand 1945, 121: 251.

8. McMenemy, W.H. Huntington's chorea. In: Greenfield, J.C., McMenemy, W.H., Norman, R.M., Blach wood, W. \& Meyer, A. (eds) Neuropathology. Edward Arnold, London, 1958, pp 475-521.

9. Lipton, M.A., Prange, A.J., Diarman, W. \& Udeufriend, S. Increased rate of norepinephine biosynthesis in hypothyroid rats. Fed Proc 1968, 27: 39.

10. Klawans, H.I. \& Shenkar, D.M. Observations on dopaminergic nature of hyperthyroid chorea. $J$ Neural Transm 1972, 33: 73-81. 